\title{
STEREO/SEPT observations of upstream particle events: almost monoenergetic ion beams
}

\author{
A. Klassen ${ }^{1}$, R. Gómez-Herrero ${ }^{1}$, R. Müller-Mellin ${ }^{1}$, S. Böttcher ${ }^{1}$, B. Heber ${ }^{1}$, R. Wimmer-Schweingruber ${ }^{1}$, and \\ G. M. Mason ${ }^{2}$ \\ ${ }^{1}$ Institut für Experimentelle und Angewandte Physik, Universität Kiel, 24118 Kiel, Germany \\ ${ }^{2}$ Applied Physics Lab, Johns Hopkins University, Laurel, MD, USA
}

Received: 17 July 2008 - Revised: 16 February 2009 - Accepted: 15 April 2009 - Published: 6 May 2009

\begin{abstract}
We present observations of Almost Monoenergetic Ion (AMI) events in the energy range of 100$1200 \mathrm{keV}$ detected with the Solar Electron and Proton Telescope (SEPT) onboard both STEREO spacecraft. The energy spectrum of AMI events contain 1, 2, or 3 narrow peaks with the relative width at half maximum of $0.1-0.7$ and their energy maxima varies for different events from 120 to $1200 \mathrm{keV}$. These events were detected close to the bow-shock (STEREO-A\&B) and to the magnetopause at STEREO-B as well as unexpectedly far upstream of the bow-shock and far away from the magnetotail at distances up to $1100 R_{E}$ (STEREO-B) and $1900 R_{E}$ (STEREO-A). We discuss the origin of AMI events, the connection to the Earth's bowshock and to the magnetosphere, and the conditions of the interplanetary medium and magnetosphere under which these AMI bursts occur. Evidence that the detected spectral peaks were caused by quasi-monoenergetic beams of protons, helium, and heavier ions are given. Furthermore, we present the spatial distribution of all AMI events from December 2006 until August 2007.
\end{abstract}

Keywords. Interplanetary physics (Energetic particles; Interplanetary shocks) - Magnetospheric physics (Energetic particles, precipitating)

\section{Introduction}

The presence of energetic ions $(<1 \mathrm{MeV})$ upstream of the Earth's bow-shock and in the Earth's magnetosheath has been well known since the 1960s (e.g. Asbridge et al., 1968). Most of such upstream bursts were observed near the bowshock at $\sim 25 R_{E}$ ( $R_{E}$, Earth's radius) (e.g. Krimigis et al.,

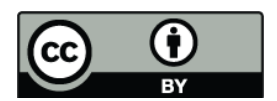

Correspondence to: A. Klassen (klassen@physik.uni-kiel.de)
1978), at the libration point (L1) at $\sim 240 R_{E}$ (Balogh et al., 1978; Scholer et al., 1981; Klassen et al., 2008), but also using STEREO observations far away from the bow-shock at the distances up to $1100 R_{E}$ (Müller-Mellin et al., 2008a; Desai et al., 2008).

The upstream ion bursts are often associated with energetic electrons $(>30 \mathrm{keV})$ and were explained in two ways: (1) by the leakage of magnetospheric particles accelerated within the magnetosphere (e.g. Sarris et al., 1978; Krimigis et al., 1978), (2) by the acceleration at the bow-shock via Fermi or shock drift acceleration (e.g. Scholer et al., 1981; Burgess 2007, and references therein).

All these mechanisms predict power-law like energy spectra with a negative slope without any spectral fine structures. Indeed such spectra were observed by different space missions during the last 40 years (e.g. Desai, 2000). Lutsenko and Kudela (1999) first reported observations of 1, 2 or 3 narrow peaks $\left(\triangle E / E_{\max } \simeq 0.15 \div 0.30\right)$ in spectra of upstream ion events using observations with the DOK-2 instrument onboard the Interball-1 spacecraft (Lutsenko et al., 1998). These ion events have been called "Almost Monoenergetic Ions" (AMI) and were observed close upstream of the bowshock and in the Earth's magnetosheath $\left(\simeq 15 R_{E}\right.$ from the nose of the bow-shock), but not in the outer magnetosphere inside of the magnetopause. Because the energy ratios in 2 and 3 peak spectra were $1: 2$ and 1:2:(5-6), respectively, they proposed that these spectral peaks can be explained as $\mathrm{H}^{+}$, $\mathrm{He}^{++}$and $\mathrm{CNO}^{(5-6)+}$ ions accelerated in a bursty, strong electrostatic field in a small region on the magnetopause or at the bow-shock (Lutsenko and Kudela, 1999; Lutsenko 2001). Occasionally the detection of a hump or a local maximum was reported near to $100 \mathrm{keV}$ in Earth's upstream events (Anderson, 1981; Anagnostopoulos et al., 2000) and in bursts upstream of the Jovian bow-shock (e.g. Zwickl et al., 1980). It has been presumed that this hump can be explained under some specific conditions either by shock drift acceleration

Published by Copernicus Publications on behalf of the European Geosciences Union. 

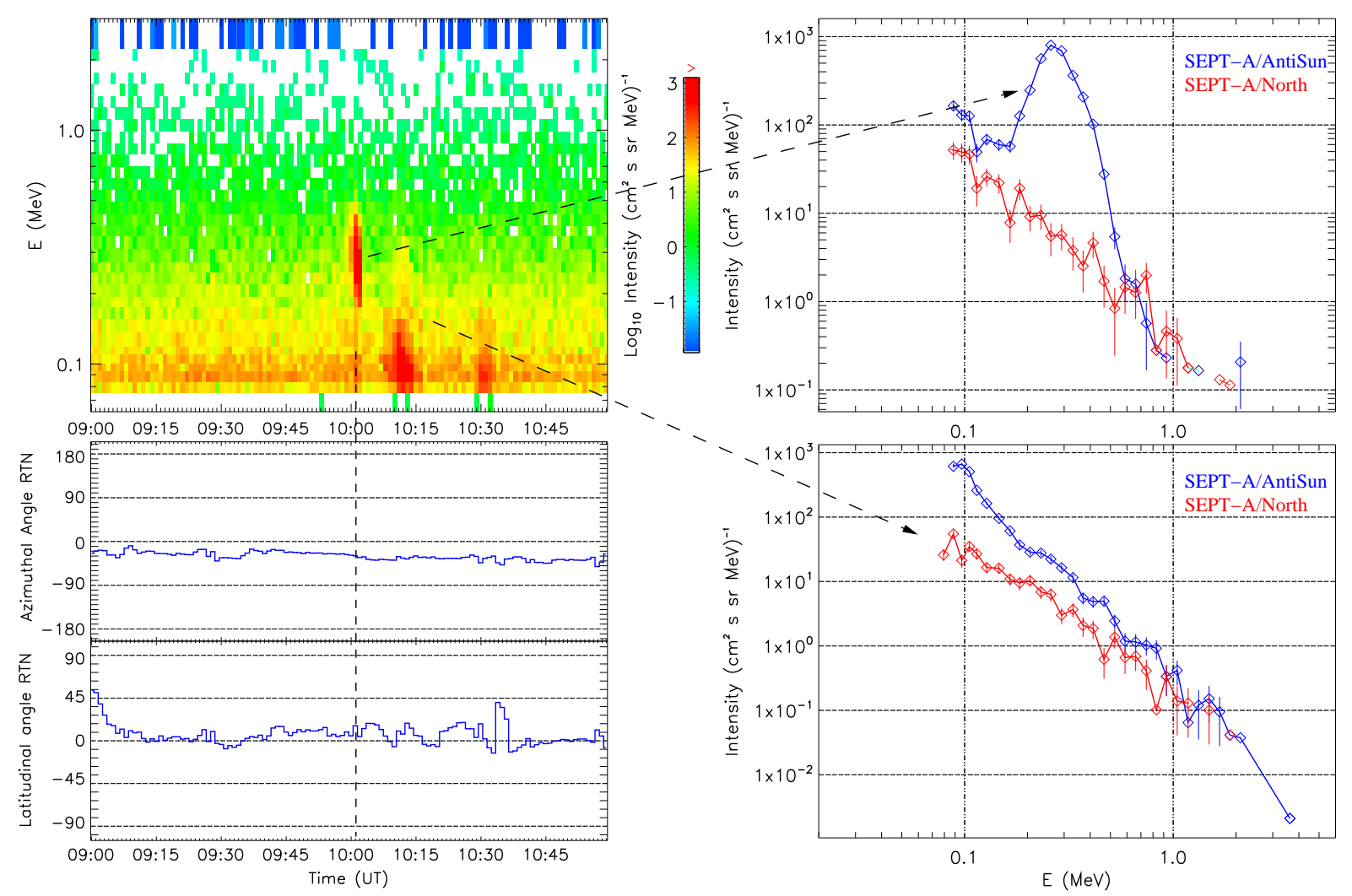

Fig. 1. Top-left, dynamic energy spectrum of an AMI event and two common upstream ion events in the range $0.1-1.2 \mathrm{MeV}$ observed with SEPT-A/Antisun far upstream of the Earth's bow-shock $\left(656 R_{E}\right)$ on 27 February 2007. Top-right, AMI spectrum with a peak at $260 \mathrm{keV}$ from SEPT-A/AntiSun (blue) and without a peak from SEPT-A/North (red) telescope. Bottom-right, for comparison the spectra of a common upstream event without any significant spectral structures. Bottom-left, the IMF components show only very small variations before and during the AMI event.

(Anderson, 1981; Decker, 1988) or by particles being accelerated in the magnetosphere and leaking into the upstream region (e.g. Anagnostopoulos et al., 2000).

The first detections of AMI events close to the bow-shock and to the magnetosphere using observations at STEREO with the SEPT instrument were already reported by MüllerMellin et al. (2008a).

In this paper we extend our observations of AMI events in the energy range of 100-1200 keV to August 2007, and to distances up to $1900 R_{E}$ and $1100 R_{E}$ from the Earth for STEREO-A\&B, respectively. We supply evidence for the acceleration mechanism and for the source of the multi-peak spectra. Furthermore, we present spatial distributions of all AMI events from December 2006 until August 2007.

\section{Instruments and data selection}

We report observations made with two instruments SEPT and SIT from the Solar Energetic Particle (SEP) suite of the STEREO IMPACT investigation. The Solar Electron and Proton Telescope (SEPT) consists of two dual double-ended magnet/foil particle telescopes which separate and measure electrons in the energy range 30-400 $\mathrm{keV}$ and ions from 60 to $7000 \mathrm{keV}$ (Müller-Mellin et al., 2008a). The energy range is divided into 32 logarithmically spaced channels with an energy resolution of $\simeq 10 \%$ in the range $\leq 1200 \mathrm{keV}$ where the AMI events were observed.

Anisotropy information on the non-spinning spacecraft is provided by two separate telescopes: SEPT-E looking in the ecliptic plane along the Parker spiral magnetic field (i.e. $45^{\circ}$ west of the spacecraft-Sun line) both towards and away from the Sun, and SEPT-NS looking vertical to the ecliptic plane towards North and South. The dual set-up refers to two adjacent sensor apertures for each of the four view directions: one for protons, one for electrons. The double-ended setup refers to the detector stack with view cones in two opposite directions: one side (electron side) is covered by a thin foil, the other side (proton side) is surrounded by a magnet. The magnet sweeps away electrons but lets ions pass. The geometry factor for each electron and proton telescope is $0.13 \mathrm{~cm}^{2} \mathrm{sr}$ and $0.17 \mathrm{~cm}^{2} \mathrm{sr}$, respectively. The SEPT field of view is $52^{\circ}$ and the time resolution is $1 \mathrm{~min}$. 
The Suprathermal Ion Telescope (SIT) is a time-of-flight mass spectrometer and is able to identify the elemental and isotopic composition of ions in the energy range from few $10 \mathrm{~s}$ of $\mathrm{keV} /$ nucleon to several $\mathrm{MeV} / \mathrm{nucleon}$. The field of view is $44^{\circ} \times 17^{\circ}$ and the boresight of the $44^{\circ}$ cone is oriented towards the Sun $60^{\circ}$ west from the spacecraft-Sun line (Mason et al., 2008). Note that SEPT measures the total energy of ions, but not its nuclear charge. Hence, the SEPT and the SIT spectra are presented on a total energy scale.

During 9 months from December 2006 until August 2007 60 AMI events were detected with SEPT using the following selection criteria (as an example see Fig. 1): a) the AMI peak is detected during two or more accumulation times (i.e. $\geq 2 \mathrm{~min}$ ), and at least in two energy channels, b) the peak maximum intensity is at least $4 \sigma$ above the pre-event background (the mean intensity is taken during $40 \mathrm{~min}$ before the event), c) the peak maximum intensity in the spectrum is two error bars higher than the intensity at the lower energy limit of the structure. Note that at energies $\leq 300 \mathrm{keV}$ the error bars are comparable with the diamond symbols in Figs. 1-5.

\section{Observations}

\subsection{Examples of AMI events}

AMI events were detected upstream and close to the bowshock at both STEREO-A\&B spacecraft as well as unexpectedly far upstream of the bow-shock at distances up to $1900 R_{E}$ (STEREO-A) and in the magnetosheath, close to the magnetopause and far away from the magnetotail at distances up to $1100 R_{E}$ (STEREO-B).

Generally, the narrow peaks were detected at different energies in the range $100-1200 \mathrm{keV}$, while the single peaks or the first peak in multi-peak events were detected below $370 \mathrm{keV}$. The events show a strong anisotropy and were only detected with telescopes directed towards the Earth, i.e. with SEPT/AntiSun telescope on STEREO-A and with SEPT/Sun telescope on STEREO-B.

Figure 1 presents an example of the dynamic energy spectrum (top-left) and the energy spectra (top-right) of an AMI event occurring on 27 February 2007 at 10:00 UT and two subsequent common ion upstream bursts observed with SEPT-A looking towards the Earth/AntiSun direction. The spacecraft was located upstream of the Earth's bowshock and $656 R_{E}$ away from the Earth (Fig. 2). The AMI event was detected during 4 successive accumulation times (4 min) and shows a narrow peak with a maximum at $E_{\max }=260 \mathrm{keV}$ and a FWHM (Full Width at Half Maximum) of $\triangle \mathrm{E}=130 \mathrm{keV}\left(\triangle \mathrm{E} / \mathrm{E}_{\max }=0.5\right)$. For comparison the following "normal" ion bursts show a simple power-law spectrum without the peak structure. It can be excluded that this narrow peak occurs due to a broken magnetic connection to the source because, at least locally close to the spacecraft, the interplanetary magnetic field (IMF) components do not show

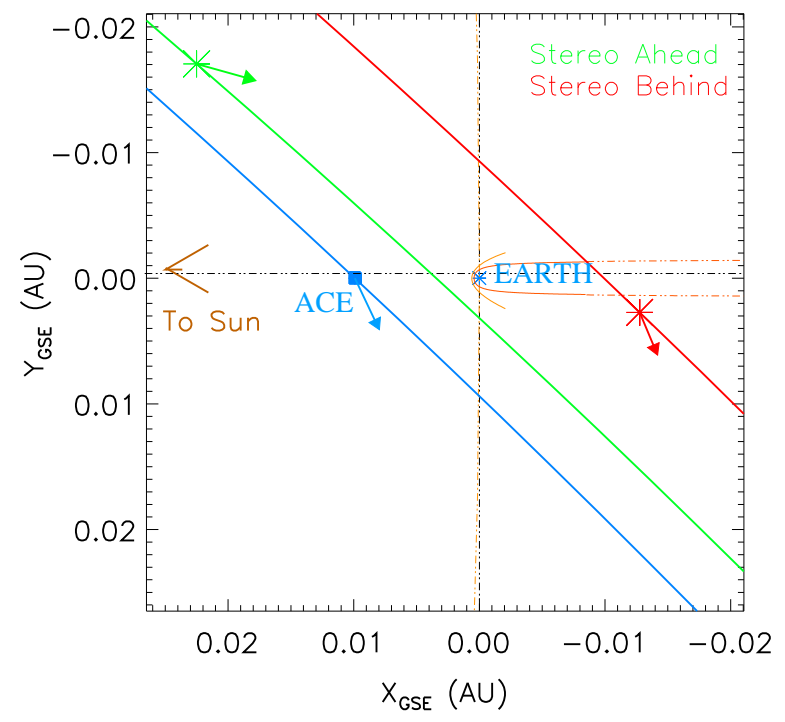

Fig. 2. Position of STEREO-A and B during the event on 27 February 2007 in relation to the Earth's bow-shock and the magnetosphere (orange lines). The arrows and the lines indicate the ecliptic IMF component and the nominal Parker spiral according to the solar wind speeds observed at both STEREO spacecraft and at ACE.

changes before, during and after the event (Fig. 1, bottomleft).

The AMI and two common events on 27 February 2007 show a strong anisotropy and they are streaming from the antisun/Earth direction, because the other three telescopes, directed towards the Sun, North, and South did not detect any enhancements (e.g. red line in Fig. 1 top-right). The vector of the IMF pointed towards the magnetosphere/magnetotail (green arrow in Fig. 2), suggesting that the observed particles indeed were streaming from the magnetosphere along the magnetic field. Figure 2 shows the position of STEREOA relative to the Earth's bow-shock and the magnetosphere during the events presented in Fig. 1.

Figure 3 shows examples of AMI events with three-peaks (a), two-peaks (b), and one-peak (c) observed with STEREO$\mathrm{B}$ when the spacecraft was at different positions far away from the magnetospheric tail (Fig. 3d).

The one-peak spectrum (Fig. 3c) was observed at a distance of $0.047 \mathrm{AU}\left(1100 R_{E}\right)$ from the Earth. During the event SEPT-B was looking towards Earth/Sun and the spacecraft was not connected to the magnetosphere or to the the bow-shock, but rather to the upstream region (red arrow indicates the ecliptic magnetic field component). For the twoand three-peak events (Fig. 3b and a) on 2 April and 6 March 2007, the distances between STEREO-B and the Earth were $656 R_{E}$ and $270 R_{E}$, respectively and the magnetic field vector pointed towards the magnetotail. The ratios of peak energies are 1:2 for two-peaks and 1:2:(5-6) for three-peak events. 

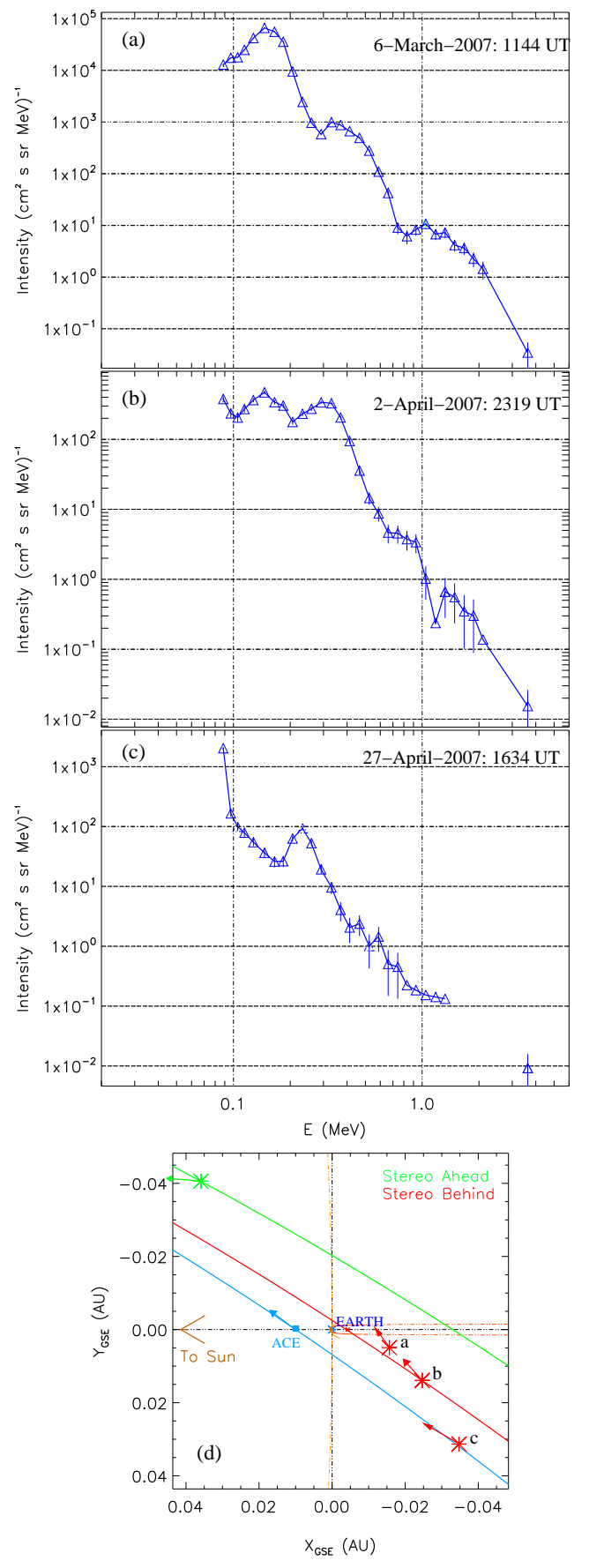

Fig. 3. AMI events observed at SEPT/STEREO-B far away from the magnetospheric tail: (a) three-peak spectrum on 6 March 2007; (b) two-peak spectrum on 2 April 2007; (c) one-peak spectrum on 27 April 2007, (d) position of STEREO-B during events a, b, c in relation to the Earth's bow-shock and the magnetosphere (orange lines). The red arrows indicate the ecliptic IMF component during the events. The red, green and blue lines show the nominal Parker spiral for event (b) according to the solar wind speeds measured at both STEREO and at ACE spacecraft, respectively.

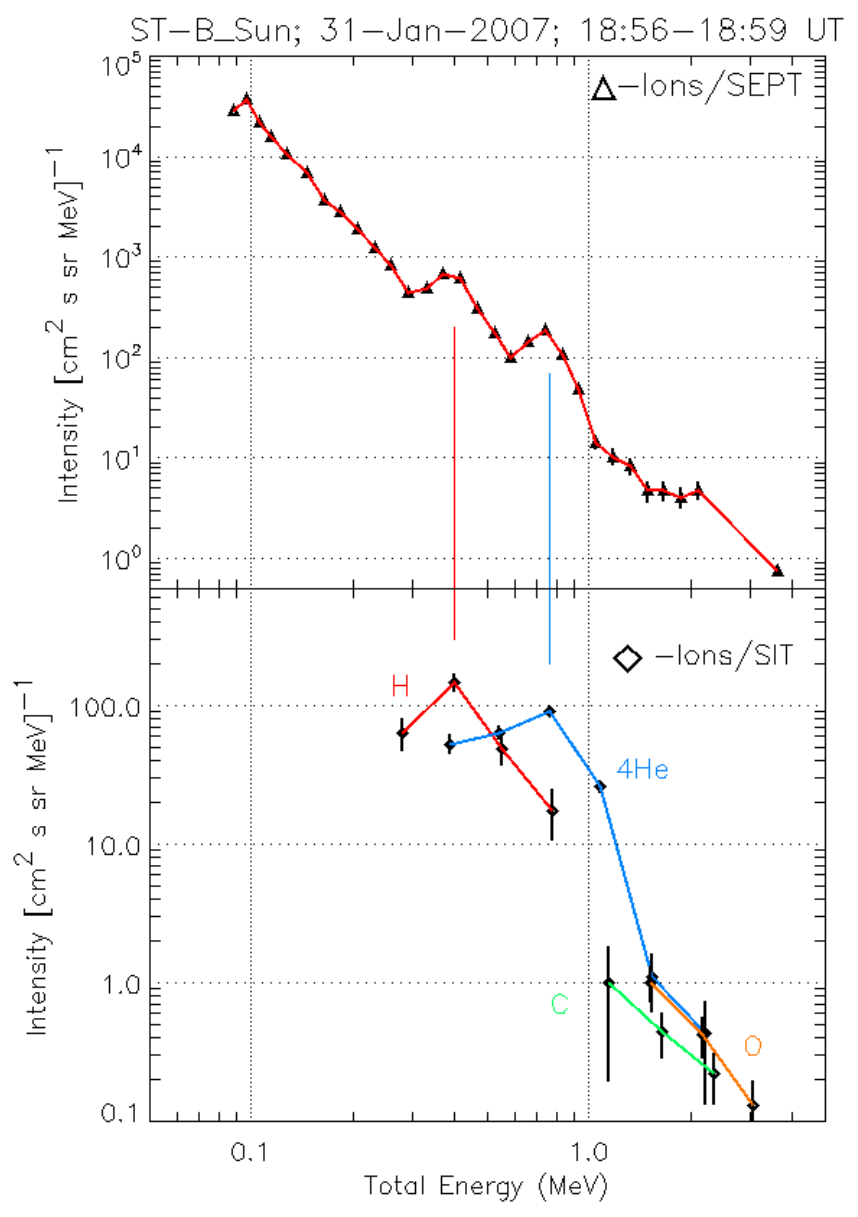

Fig. 4. AMI event observed simultaneously with the SEPT (top panel) and SIT (bottom panel) instruments onboard STEREO-B on 31 January 2007. Both spectra show two peaks around 370 and $740 \mathrm{keV}$ with the peak energy ratio of 1:2.

The association between AMI events and electron bursts is weak. Only during 9 of 60 AMI events a weak electron burst at energies $45-100 \mathrm{keV}$ was nearly simultaneously ( $\pm 2 \mathrm{~min}$ ) observed. The electron spectra do not contain any spectral peaks and show a usual power-law shape. We suppose that these electrons are not really associated with the ion increases and are only chance coincidences. Indeed, if both types of particles had escaped from the same source at the same time, the time delay between electrons and protons on arrival should be between 3 and $36 \mathrm{~min}$, due to velocity dispersion between electrons and ions and due to progressive increase of the distance between the spacecraft and the Earth. This supports the observations of Lutsenko and Kudela (1999) reporting a total absence of energetic electron fluxes exceeding background levels during AMI events. 

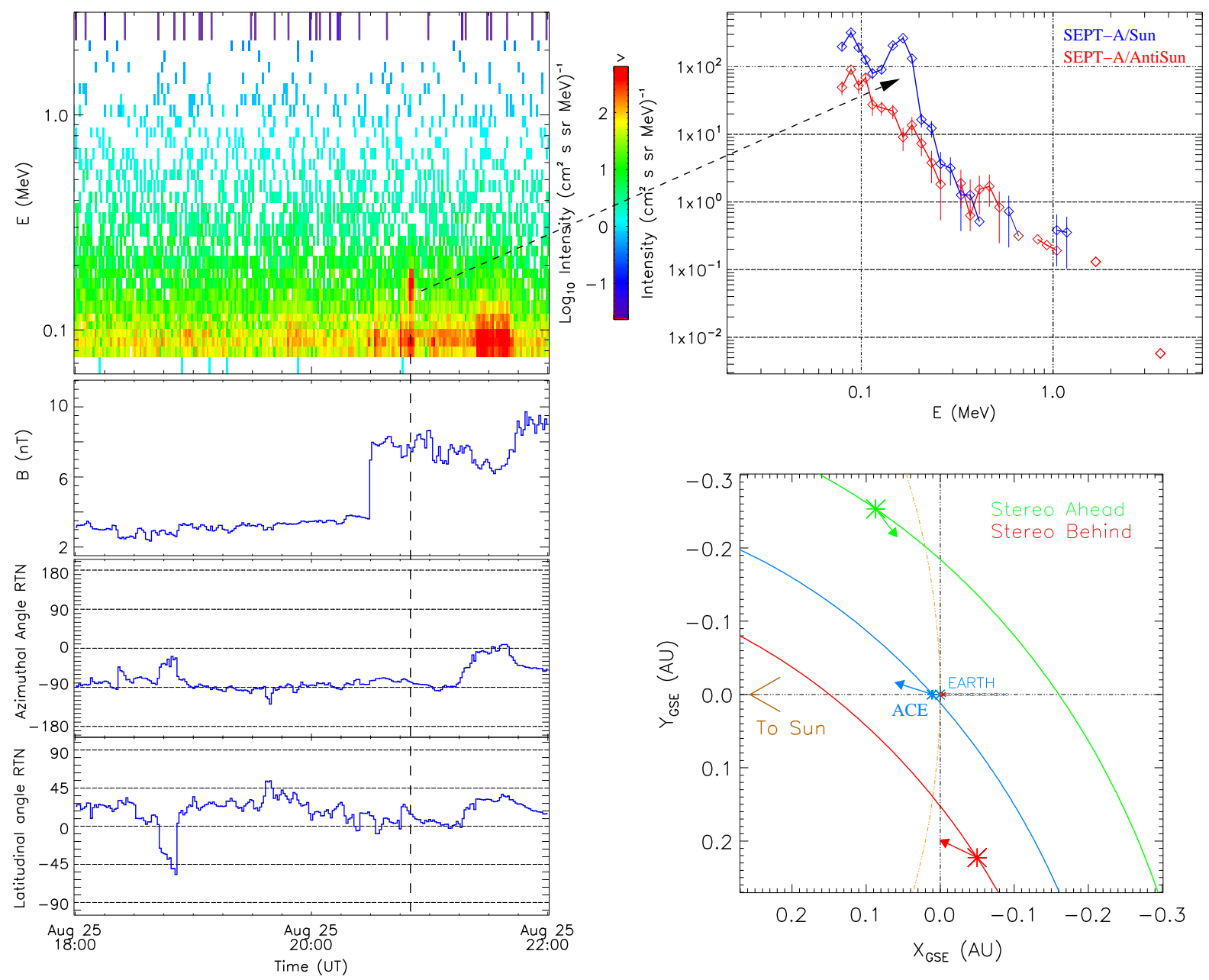

Fig. 5. AMI event on 25 August 2007 detected from the AntiEarth/Sun direction. It occurs 20 min after the passage of a CIR forward shock (second panel). Top-right, spectrum with a peak at $165 \mathrm{keV}$ from SEPT-A/Sun (blue) and without a peak from SEPT-A/AntiSun telescope. Bottom-right, the position of STEREO-A (green) and the IMF ecliptic component during the event (arrow).

\subsection{Comparison of AMI events observed with the SEPT and SIT}

Lutsenko and Kudela (1999) suggested that due to the peak energy ratios in two and three-peak spectra as 1:2:(5-6) the AMI events could be explained as $\mathrm{H}^{+}, \mathrm{He}^{++}$and $\mathrm{CNO}^{(5-6)+}$ ions accelerated in a burst of electrostatic field. In order to explore direct evidence for this idea we compared AMI spectra observed simultaneously with the SEPT and SIT instruments at STEREO-B during the event on 31 January 2007. In contrast to SEPT, the SIT instrument is able to distinguish between different species, e.g. H, He and CNO.

Figure 4 presents this comparison. We note that the absolute intensities reported by SEPT and SIT are not the same due to differing view cones, and uncertainties in the detection efficiency for protons by SIT. There are two distinct narrow peaks in SEPT and SIT spectra which match each other very well. The SEPT spectrum shows two peaks with max- ima around 370 and $740 \mathrm{keV}$ and the SIT instrument detects the first peak in $\mathrm{H}$ spectrum at $400 \mathrm{keV}$ and the second peak in $\mathrm{He}$ at $760 \mathrm{keV}$ total energy. The peak maximum ratio is close to 1:2 for both instruments which could be explained by accelerated solar wind $\mathrm{H}^{+}$and $\mathrm{He}^{++}$ions. In addition there is a small hump around $1200 \mathrm{keV}$ in the SEPT spectrum, which could be associated with enhanced counts of solar wind $\mathrm{CO}^{(5-6)+}$ ions. Unfortunately, this hump is not resolved in SIT CO spectra, most likely due to poor counting statistics. During the event STEREO-B was $115 R_{E}$ away from the Earth, close to the magnetopause, at GSE coordinates: $X_{\mathrm{GSE}}=-94 R_{E}, Y_{\mathrm{GSE}}=+47 R_{E}$ and $Z_{\mathrm{GSE}}=+48 R_{E}$.

\subsection{AMI events associated with a CIR shock}

Almost all AMI events observed at STEREO-A were detected with the SEPT-A/Antisun telescope revealing a strong anisotropy of particles streaming from the Earth (antisun) 

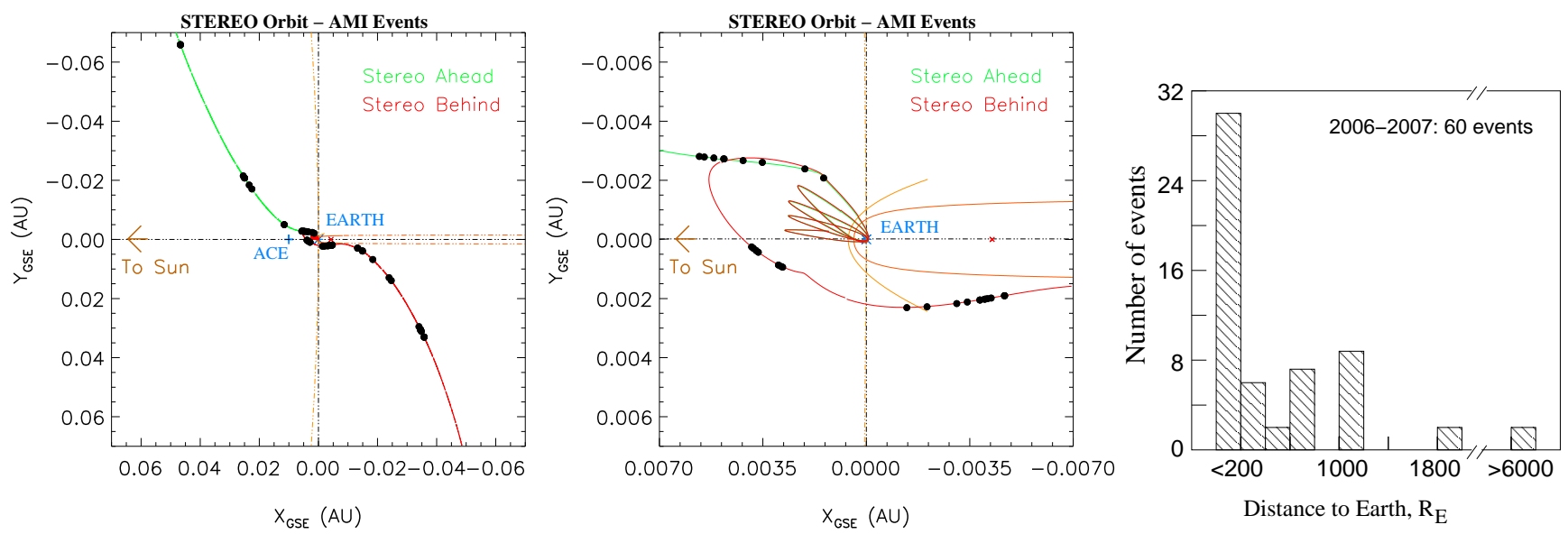

Fig. 6. The spatial distribution of AMI events observed along the both STEREO-A\&B orbits (green and red lines) during the time interval from 15 December 2006 to 29 April 2007. Each event (black point) are shown in relation to the Earth's bow-shock and the magnetopause (orange lines). The Sun is to the left. The right panel presents the number of AMI events as a function of radial distance from the Earth. Predominately, the AMIs occur at distances smaller than $200 R_{E}$, but there are still events observed further than $1800 R_{E}$. The two events near $6000 R_{E}$ are associated with the passage of a IP shock.

direction. That is expected if the source of AMIs is located close to the Earth. Two exceptional events were observed on 25 August 2007 with the SEPT-A/Sun telescope, suggesting that these beams stream from the Sun direction. However, they were not associated with any activity at the Sun.

Figure 5 shows one of them when STEREO-A was $0.27 \mathrm{AU}\left(6330 R_{E}\right)$ away from the Earth. The top-left figure presents the dynamic spectrum of the AMI event at 20:50 UT followed by a small normal event. The top-right figure presents the AMI spectra from the sunward (blue) and from the antisunward (red) directions. The blue one contains a narrow peak at $165 \mathrm{keV}$, while the red one shows a powerlaw spectrum without significant structures. The subsequent event at 21:30 UT shows a usual power-law spectrum without distinct anisotropy. As the AMI event occurs 20 min after a shock passage (Fig. 5, bottom-left) and as the IMF ecliptic components during and before the event do not show significant changes, it is suggested that this event is associated with the shock passage and is not an upstream event originating somewhere near to the Earth.

Nevertheless, we can not totaly exclude that the spacecraft was connected to the Earth after all, if the magnetic field strongly twist around. Indeed, Stansberry et al. (1988) showed on ISEE-3 that local measurement of the IMF is not a reliable indicator of connection to the Earth. Desai et al. (2008) also confirmed this and suggested that upstream events were seen in connection with large-scale $(0.03 \mathrm{AU})$ Alfven waves which facilitate the transport of upstream ions from the bow-shock. In our opinion such scenario is not very probable for this case, because the spacecraft was already 0.27 AU away from the Earth.

\subsection{Spatial distribution of AMI events}

Both STEREO-A\&B spacecraft detected altogether 60 AMI events at different times and positions. The AMI's occur not only upstream and close to the Earth's bow-shock, but also far away from the Earth up to distances of $0.081 \mathrm{AU}$ $\left(1900 R_{E}\right)$ and $0.049 \mathrm{AU}\left(1148 R_{E}\right)$ on STEREO-A\&B, respectively (Fig. 6). Furthermore, at STEREO-B the AMI events were detected also inside the magnetosheath, close to the magnetopause (Fig. 6, middle), and far away from the magnetotail. In the last case the observed AMI events do not represent the usual upstream events, but possibly a new class of events, because they were observed not upstream of the bow-shock. These events may not be associated with the Earth's bow-shock and its upstream region.

The event distribution along the orbit of both spacecraft is not uniform and shows a distinct clustering, which reflects an association with solar wind high-speed streams and enhanced geoactivity (e.g. Anagnostopoulos et al., 2000; Desai et al., 2000) rather than with the connectivity to the bowshock and to the magnetosphere as for usual upstream events. The whole event distribution of number of events vs. distance is presented in Fig. 6 (right). About $50 \%$ of the events occur at distances $\leq 200 R_{E}$. Two events around $6000 R_{E}$ are associated with a CIR shock passage and were presumably not of magnetospheric origin (see Sect. 3.3).

\subsection{Summary of observational results}

The SEPT instruments on board both STEREO spacecraft detected AMI events whose energy spectra contain one, two, or three narrow peaks with mean $\triangle \mathrm{E} / \mathrm{E}_{\max }=0.4$. The main properties of $60 \mathrm{AMI}$ events detected in the energy range 
0.1-1.2 MeV, observed from December 2006 to August 2007 are presented in Fig. 7a-d. The observational results of AMI's are the following:

1. AMI events were observed not only close to or far upstream of the bow-shock and inside the magnetosheath, but also far away from the Earth up to distances of $0.081 \mathrm{AU}\left(1900 R_{E}\right)$ and $0.049 \mathrm{AU}\left(1148 R_{E}\right)$ on STEREO-A\&B, respectively. STEREO-A detected only one-peak spectra, while on STEREO-B spectra with one as well as with two or three peaks were observed. The multi-peak events were observed when STEREO-B was connected with the magnetopause/magnetotail, only. The distribution of peaks number is shown Fig. 7a.

2. On average the AMI duration is $4.3 \mathrm{~min}$ and lies in the range from few to $30 \mathrm{~min}$. Some events with durations longer than 10 min occurred in groups (Fig. 7b).

3. The ratios of the peak energies for spectra with 2 or 3 peaks are about 1:2:(5-6). This may be explained as acceleration of $\mathrm{H}^{+}, \mathrm{He}^{++}$and $\mathrm{CNO}^{(5-6)+}$ ions in a burst of electrostatic field to energies proportional to their charges Q (Lutsenko and Kudela, 1999).

4. Comparison of SEPT and SIT observations undoubtedly support the idea that the multi-peak spectral structures reflect the presence of monoenergetic beams of $\mathrm{H}, \mathrm{He}$ and $\mathrm{CNO}$ ions (Fig. 4).

5. The relative FWHM of the peaks varied between 11$75 \%$ with a mean of $40 \%$ (Fig. $7 d$ ).

6. Only two AMI events streaming from the Sun/antiEarth direction were detected with SEPT-A at distances $0.27 \mathrm{AU}$ from the Earth and both were associated with the passage of a forward CIR shock. This suggests that the AMI generation process may also act at IP shocks or, what is less probable, the IP magnetic field strongly twists around, so that the event was detected from the unexpected "wrong" antiEarth direction.

7. Only $15 \%$ of 60 AMI events were observed about simultaneously ( $\pm 2 \mathrm{~min}$ ) together with $45-100 \mathrm{keV}$ electrons. However, these electron events do not display any peaks in their spectra. We suppose that these electrons are not really associated with the ion increases and are only chance coincidences, because the electrons should arrive always sooner due to velocity dispersion (see Sect. 3.1).

8. No obvious IMF structures (e.g. Fig. 1) coincides with the majority of AMI events, suggesting they are true isolated events and can not be explained as a consequence of dispersive propagation with intermittent connection to the source.
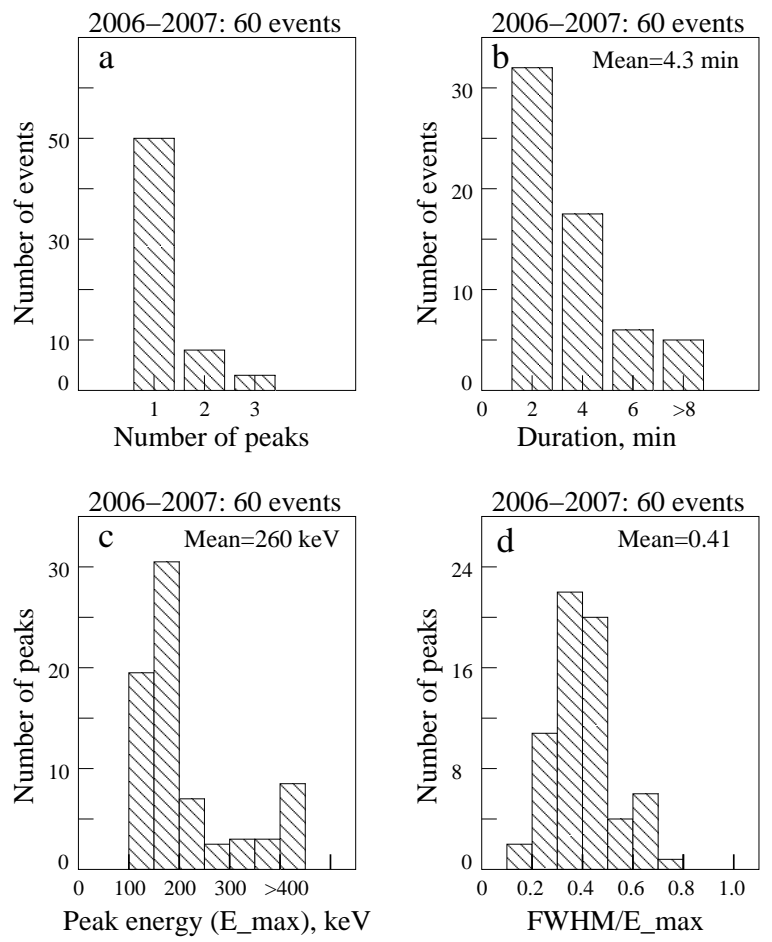

Fig. 7. Distributions of AMI characterictics. (a) Number of peaks in spectra. In the majority of events (50 of 60) only one peak is detected. (b) Duration of AMI events. The mean duration is $4.3 \mathrm{~min}$. Only events with duration $\geq 2 \mathrm{~min}$ are included in the statistics. (c) Distribution of peak energy and (d) the relative width

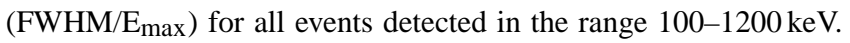
The majority of peaks occur at energies $\leq 200 \mathrm{keV}$ with relative FWHM $\leq 0.4$. The mean values of the distributions are given in the plots.

\section{Discussion}

Observation of narrow peaks in spectra of ions streaming from Earth's bow-shock, magnetosphere or from a CIR shock suggests that they are quasi-monoenergetic beams of ions accelerated at the bow-shock, CIR shock or accelerated inside the magnetosphere/magnetopause and then ejected into interplanetary space. The peak energy ratios in multipeak spectra as 1:2:(5-6) can be explained as $\mathrm{H}^{+}, \mathrm{He}^{++}$and $\mathrm{CNO}^{(5-6)+}$ ion acceleration in a burst of electrostatic field to energies proportional to the charges $\mathrm{Q}$ of these ions as proposed by Lutsenko and Kudela (1999) because all other known acceleration mechanisms predict a power-law like energy spectrum without narrow spectral structures. It should be noted, that under some specific conditions, it was proposed that an occasionally observed hump near $100 \mathrm{keV}$ can be interpreted as a signature of shock drift acceleration (Anderson, 1981; Decker, 1988) or as a leakage of magnetospheric particles into the upstream region due to rigiditydependent escape (Anagnostopoulos et al., 2000). 
The last two mechanisms can under specific conditions produce one-peak spectra, but it is not clear how they can explain two- and three-peak spectra, in particular the peak energy ratio of 1:2:5-6. Furthermore, a lot of 1-3 peak AMI events were observed at STEREO-B, when the spacecraft was downstream of the bow-shock or close/far to the magnetotail. In these regions the shock drift acceleration predicts near power-law distributions without peaks or humps (e.g. Decker, 1988).

The idea of quasi monoenergetic beams of ions accelerated in electrostatic fields can explain these features and is strongly supported by the comparison of SEPT and SIT observations of narrow peaks in $\mathrm{H}$ and $\mathrm{He}$ spectra (Fig. 4). The main problem of this idea is to explain the origin of electrostatic fields in different places/sources, e.g. near bow- and CIR-shocks, and near the magnetosphere/magnetopause (but see Lutsenko, 2001).

The AMIs spectra can not be explained as a consequence of velocity dispersion and due to a short connection to the source even when the particles had a continuous power-law spectrum in the source. That is clear from the following simple reasoning. For instance, the two-peak spectra of $\mathrm{H}^{+}$and $\mathrm{He}^{++}$were detected simultaneously (time resolution $1 \mathrm{~min}$ ) and their onset time should be determined as:

$t_{\text {onset }}^{H}=t_{\text {onset }}^{H e}=\frac{L}{V_{H}}=\frac{L}{V_{H e}}$

where $V$ is the velocity of ions and $L$ is the path length from the source to the spacecraft. Assuming $L$ is equal for both species, we get $E_{H e} / E_{H}=4$, but the observed ratio of $E_{H e} / E_{H}$ is 2 . Therefore the detected spectral peaks are really narrow peaks in the acceleration source, too.

The trajectories of STEREO-A and STEREO-B are very different in relation to the magnetosphere and bow-shock. While STEREO-A ahead of the Earth could be mainly magnetically connected to the bow-shock, STEREO-B was a short time in the upstream region, but most of the time close to the magnetopause and then further away from the magnetotail region (see Fig. 6), i.e. it could be connected with these regions. Note, it is a big challenge to extrapolate the magnetic field connections to the source particularly at such large distances. In fact, small changes in the direction of IMF can strongly affect the magnetic connection (e.g. Giacalone et al., 2000).

Nevertheless, it is plausible to suppose that the AMI events originating in different sources should demonstrate also some different properties. Indeed, there is a difference in ocurrence of one-peak and multi-peak AMI events observed respectively by STEREO-A and STEREO-B. Upstream of the bow-shock, both spacecraft observed one-peak spectra only. Multi-peak spectra were observed only by STEREO$\mathrm{B}$ when it was rather connected to the magnetopause in the magnetotail region. Therefore, we propose that the acceleration mechanisms acting in these two sources are slightly different. Another hint towards the acceleration mechanism modification is provided by the observation of the one-peak spectra on 25 August 2007. These particles were streaming from the Sun direction and not from the Earth. They were associated with a CIR forward shock being convected over the spacecraft (Fig. 5).

Why SEPT-A did not detect multi-peak spectra even though it was sometimes connected to the magnetotail is not clear yet. A simple explanation is based on low statistic of events observed with STEREO-A (only 18 AMI events), because at Interball-1 the multi-peaks were also observed upstream of the bow-shock. Another possible explanation is that the heavier ions of $\mathrm{He}^{++}$and $\mathrm{CNO}^{(5-6)+}$ accelerated somewhere inside the magnetosheath/magnetopause were modified by their passage through the bow-shock front so that only protons could pass the shock or that the composition of $\mathrm{H}, \mathrm{He}$, and $\mathrm{CNO}$ are different in these regions. An alternative interpretation, is that the heavier ions may be more strongly influenced/affected by some kind of turbulence/wave activity during their passage through the foreshock region (Desai et al., 2008). How all these mechanisms work in detail is not clear now, but, it appears from our observations that the particles mass/charge ratio may play a crucial role.

In general, the detection of upstream events far away from the Earth by STEREO-B suggests that their origin can be explained in terms of leakage of magnetospheric particles rather than by Fermi acceleration at the Earth's bow-shock, because the spacecraft was well connected to the magnetospheric tail or sometimes to the dusk sector of the bow-shock and not to the quasi-parallel part of the bow-shock.

The events detected at the location of STEREO-B close to and far from the tail region of the magnetosphere possibly represent a new class of bursts - magnetospheric particle events (jets) (Gómez-Herrero et al., 2009) - which are not related to the shock upstream region.

\section{Conclusions}

We have presented observations of Almost Monoenergetic Ion (AMI) events containing 1-3 narrow peaks in their energy spectra. We attribute the peaks to quasi monoenergetic beams of $\mathrm{H}^{+}, \mathrm{He}^{++}$and $\mathrm{CNO}^{(5-6)+}$ accelerated at the shock, at the magnetopause or inside the magnetosheath in a burst of electrostatic field to energies proportional to their charges Q as proposed by Lutsenko and Kudela (1999). The AMI events were detected not only close to and upstream of the Earth's bow-shock but also far away from the magnetosphere up to distances of $1900 R_{E}$. Additionally we have presented observations of AMI events associated with the passage of a CIR forward shock. We suggest that the bow-shock and the CIR-shock accelerate predominantly AMI's with one-peak spectra, while the acceleration mechanism acting at the magnetopause or/and inside the magnetosheath is able 
to accelerate quasi monoenergetic beams of $\mathrm{H}^{+}, \mathrm{He}^{++}$and $\mathrm{CNO}^{(5-6)+}$ ions.

Acknowledgements. This work was supported by Deutsches Zentrum für Luft und Raumfahrt (DLR) under grants No. 50 OC 0105 and No. 50 OC 0004. The authors thank two anonymous referees for their comments and suggestions.

Topical Editor I. A. Daglis thanks two anonymous referees for their help in evaluating this paper.

\section{References}

Anagnostopoulos, G. C., Paschalidis, N., and Littas, A. N.: Energy time dispersion of a new class of magnetospheric ion events observed near the Earth's bow shock, Ann. Geophys., 18, 28-41, 2000, http://www.ann-geophys.net/18/28/2000/.

Anderson, K. A.: Measurements of bow shock particles far upstream from the Earth, J. Geophys. Res., 86, 4445-4454, 1981.

Asbridge, J. R., Bame, S. J., and Strong, I. B.: Outward flow of protons from the Earth's bow-shock, J. Geophys. Res., 73, 57775782, 1968.

Balogh, A., Hynds, R. J., van Rooijen, J. J., Stevens, G., Domingo, V., Sanderson, T. R., and Wenzel, K.-P.: Evidence for magnetosphere proton bursts: First results from the low energy proton experiment on ISEE 3 (abstract), Eos Trans. AGU, 59, 1174, 1978.

Burgess, D.: Particle acceleration at the Earth's bow-shock, Lect. Notes Phys. 725, 161-190, 2007.

Giacalone, J., Jokipii, J. R., and Mazur, J. E.: Small-scale gradients and large-scale diffusion of charged particles in the heliospheric magnetic field, Astrophys. J, 532, 75-78, 2000.

Gómez-Herrero, R., Klassen, A., Müller-Mellin, R., Heber, B., Wimmer-Schweingruber, R., and Böttcher, S.: Recurrent CIRaccelerated ions observed by STEREO SEPT, J. Geophys. Res., 114, A05101, doi:10.1029/2008JA013755, 2009.

Decker, R. B.: Computer modeling of test particle acceleration at oblique shocks, Space Sci. Rev., 48, 195-262, 1988.

Desai, M. I., Mason, G. M., Müller-Mellin, R., Korth, A., Dwyer, J. R., and von Rosenwinge, T. T.: The spatial distribution of upstream ion events from the Earth's bow shock measured by ACE, Wind, and STEREO, J. Geophys. Res., 113, A08103, doi:10.1029/2007JA012909, 2008.

Desai, M. I., Mason, G. M., Dwyer, J. R., Mazur, J. E., von Rosenvinge, T. T., and Lepping, R. P.: Characteristics of energetic ( $\geq 30 \mathrm{keV} /$ nucleon) ions observed by the Wind/STEP instrument upstream of the Earth's bow-shock, J. Geophys. Res., 105, 61-78, 2000.
Klassen, A., Gómez-Herrero, R., Böhm, E., Müller-Mellin, R., Heber, B., and Wimmer-Schweingruber, R.: COSTEP/SOHO observations of energetic electrons far upstream of the Earth's bow-shock, Ann. Geophys., 26, 905-912, 2008, http://www.ann-geophys.net/26/905/2008/.

Krimigis, S. M., Venkatesan, D., Barichello, J. C., and Sarris, E. T.: Simultaneous measurements of energetic protons and electrons in the distant magnetosheath, magnetotail, and upstream in the solar wind, Geophys. Res. Lett., 5, 961-964, 1978.

Lutsenko, V. N. and Kudela, K.: Almost monoenergetic ions near the Earth's magnetosphere boundaries, Geophys. Res. Lett., 26, 413-416, 1999.

Lutsenko, V. N.: Almost Monoenergetic Ions: New support for Alfven Ideas on the Role of Electric Currents in Space Plasmas, Asbridge, J. Phys. Chem. Earth (C), 26, 615-619, 2001.

Lutsenko, V. N., Kudela, K., and Sarris, E. T.: The DOK-2 experiment to study energetic particles by Tail Probe and Auroral Probe satellites in the Interball project, Cosmic Research, 36, 93-102, 1998.

Mason, G. M., Korth, A., Walpole, P. H., Desai, M. I., von Rosenvinge, T. T., and Shuman, S. A.: The Suprathermal Ion Telescope (SIT) for the IMPACT/SEP Investigation, Space Sci. Rev., 136, 257-284, doi:10.1007/s11214-006-9087-9, 2008.

Müller-Mellin, R., Gómez-Herrero, R., Böttcher, S., Klassen, A., Heber, B., Wimmer-Schweingruber, R., Duvet, L., and Sanderson, T. R.: Upstream events and recurrent CIR-accelerated particle events observed by Stereo/SEPT, Proc. of the 30th ICRC, edited by: Caballero, R., D’Olivo, J. C., Medina-Tanco, G., Nellen, L., Sánchez, F. A., and Valdés-Galicia, J. F., Mexico, 2008, vol. 1, pp. 371-374, 2008a.

Müller-Mellin, R., Böttcher, S., Falenski, J., Rode, E., Duvet, L., Sanderson, T. R., Butler, B., Johlander, B., and Smit, H.: The Solar Electron and Proton Telescope for the STEREO Mission, Space Sci. Rev., 136, 363-389, 2008b.

Sarris, E. T., Krimigis, S. M., Bostrom, C. O., and Armstrong, T. P.: Simultaneous multispacecraft observations of energetic proton bursts inside and outside the magnetosphere, J. Geophys. Res., 83, 4289-4305, 1978.

Scholer, M., Hovestadt, D., Ipavich, F. M., and Gloeckler, G.: Upstream energetic ions and electrons: Bow shock-associated or magnetospheric origin?, J. Geophys. Res., 86, 9040-9046, 1981.

Stansberry, J. A., Gosling, J. T., Thomsen, M. F., Bame, S. J., and Smith, E. J.: Interplanetary magnetic field orientations associated with bidirectional electron heat fluxes detected at ISEE 3, J. Geophys. Res., 93, 1975-1980, 1988.

Zwickl, R. D., Krimigis, S. M., Armstrong, T. P., and Lanzerotti, L. J.: Ions of Jovian origin observed by Voyager 1 and 2 in interplanetary space, Geophys. Res. Lett., 7, 453-456, 1980. 